\title{
Stability Analysis of a Class of Higher Order Difference Equations
}

\author{
Yuanyuan Liu ${ }^{1}$ and Fanwei Meng ${ }^{2}$ \\ ${ }^{1}$ Department of Mathematics, Shanghai Normal University, Shanghai 200234, China \\ ${ }^{2}$ Department of Mathematics, Qufu Normal University, Qufu 273165, China
}

Correspondence should be addressed to Yuanyuan Liu; liuyuanyuan1117@163.com

Received 31 March 2014; Accepted 6 May 2014; Published 28 May 2014

Academic Editor: Yonghui Xia

Copyright (c) 2014 Y. Liu and F. Meng. This is an open access article distributed under the Creative Commons Attribution License, which permits unrestricted use, distribution, and reproduction in any medium, provided the original work is properly cited.

We consider the sufficient conditions for asymptotic stability and instability of certain higher order nonlinear difference equations with infinite delays in finite-dimensional spaces. With the aid of the general comparison condition on the right-hand side function $f_{k}(\cdot)$, we generalize the stability and instability result.

\section{Introduction}

In the field of statistics, people get used to mark the number of sample as discrete parameter not continuous for applied meaning. There are some discrete models in real life such as cobweb model of demand and supply and national income model, which could not be described by differential equation under the factual conditions. Then difference equations arise and are widely used in areas such as information transmission and population models [1-4].

During the period of development of mathematics together with information revolution, there are many discrete dynamic systems to describe the real problem, such as the monographs $[5,6]$. For example, the book Liber Abaci, written by the famous mathematician Leonardo di Pisa, stated the so-called Fibonacci sequence to the problem of rabbits' number. To march the development of mathematic discrete models in application, difference equations raised the form of higher orders or with delays; see [7-9]. Besides, the stability of the equilibrium is always a great topic to study, such as [10-13] and the references therein. Also, there are some researchers engaged in studying the stability analysis of the equations with delay; see $[14,15]$.

As the simplest case, the first order difference equation

$$
y_{k+1}=f_{k}\left(y_{k}\right), \quad k \geq 0 \in Z,
$$

has been paid much attention to and widely investigated in the literature.
In this paper, we consider a higher order difference equation with delays

$$
y_{k+1}=f_{k}\left(y_{k}, y_{k-1}, \ldots, y_{k-r}, \sum_{s=0}^{k-1} g_{k, s}\left(y_{s}, y_{s-1}, \ldots, y_{s-r+1}\right)\right)
$$

where $y_{k} \in X, X$ is a Banach space, and $f_{k}(\cdot), g_{k, s}(\cdot)$ are functions on $Z \times X^{r+1} \rightarrow X$ and $Z^{2} \times X^{r-1} \rightarrow X$, respectively. We assume that (2) satisfies the initial condition $\left(x_{0}\right.$, $\left.x_{-1}, \ldots, x_{-r}\right)$, which means that the solution of system (2), $\left\{y_{n}\right\}_{n \geq-r}$, holds for $y_{i}=x_{i}, i=-r, 1-r, \ldots, 0$. In addition, we suppose that the zero solution is the unique equilibrium; in other words, $f_{k}(c, c, \ldots, c)=c$ for some real values and all $k \in Z^{+}=\{x \mid x \geq 0, x \in Z\}$ if and only if $c=0$. Yao and Meng in [9] studied the similar system and gave some sufficient conditions to guarantee that the solution satisfies a contained equation under the method of a discrete inequality. We note that the system (2) always has solution at the time $k$ given by

$$
\begin{aligned}
& y_{1}=f_{0}\left(y_{0}, y_{-1}, \ldots, y_{-r}\right), \\
& y_{2}=f_{1}\left(y_{1}, y_{0}, \ldots, y_{1-r}, g_{1,0}\left(y_{0}, y_{-1}, \ldots, y_{-r}\right)\right),
\end{aligned}
$$




$$
\begin{aligned}
y_{k}=f_{k-1}\left(y_{k-1}, y_{k-2}, \ldots, y_{k-r-1},\right. \\
\left.\quad \sum_{s=0}^{k-2} g_{k-1, s}\left(y_{s}, y_{s-1}, \ldots, y_{s-r+1}\right)\right) .
\end{aligned}
$$

It is easy to see that $y_{k}$ is defined by the solution $y_{k-1}$.

The main aim of this paper is to provide the sufficient conditions for asymptotic stability and instability of (2). First, we state some notations and basic definitions used in the paper. Denote that $\|\mathbf{x}\|=\sqrt{x_{0}^{2}+x_{1}^{2}+\cdots+x_{n}^{2}}$ for the vector $\mathbf{x}=\left(x_{0}, x_{1}, \ldots, x_{n}\right) \in X$, which implies that, for $f, g \in X$,

$$
\|f \cdot g\| \leq\|f\| \cdot\|g\|, \quad\|f+g\| \leq\|f\|+\|g\| .
$$

Definition 1. The zero solutions of the system (2) are stable if, for every $\varepsilon>0, k_{0} \in Z^{+}$, there exists $\delta\left(\varepsilon, k_{0}\right)>0$ such that every solution $\left\{x_{k}\right\}$ of the system with the initial condition $\left\|\left(x_{k_{0}-k}, \ldots, x_{k_{0}}\right)\right\| \leq \delta$ satisfies the condition $\left\|x_{k}\right\|<\varepsilon$, for all $k \geq k_{0}$.

Definition 2. The zero solutions of the system (2) are asymptotically stable if it is stable and, under a constant $\mu$ existing, $\lim _{k \rightarrow \infty}\left\|x_{k}\right\|=0$ for any solution $\left\{x_{n}\right\}$ of (2) such that $\left\|\left(x_{k_{0}-k}, \ldots, x_{k_{0}}\right)\right\| \leq \mu$.

Definition 3. The zero solutions of system (2) are unstable if it is not stable, that is, if there are numbers $\varepsilon>0, k_{0} \in Z^{+}$, such that for every $\delta>0$ there is a solution $\left\{x_{k}\right\}$ with the initial condition $\left\|\left(x_{k_{0}-k}, \ldots, x_{k_{0}}\right)\right\| \leq \delta$ and there exists a number $K \geq k_{0}$ such that $\left\|x_{K}\right\| \geq \varepsilon$.

The system we consider is a general form of higher order difference equations without the concrete formula of $f_{k}(\cdot)$. If we entitle the function $f_{k}(\cdot)$ to a special form, such as the form of logistic function, then the stability of equilibrium points can be used to discuss the population model.

\section{Asymptotic Stability}

In this section, using the method of comparison conditions, we will obtain the sufficient conditions of the nonlinear difference system (2). The following discrete inequalities we assumed play an important role in the investigations of the asymptotic stability:

$$
\begin{array}{r}
\left\|f_{k}\left(x_{0}, x_{1}, \ldots, x_{r+1}\right)\right\| \leq \alpha \prod_{i=0}^{r}\left\|x_{i}\right\|^{p_{i}}\left\|x_{r+1}\right\|, \\
\alpha>0, \quad p_{i} \geq 0, \quad i=0,1, \ldots, r
\end{array}
$$

$$
\begin{gathered}
\left\|g_{k, s}\left(x_{0}, x_{1}, \ldots, x_{r-1}\right)\right\| \leq \beta \prod_{i=1}^{r}\left\|x_{i-1}\right\|^{q_{i}}, \\
\beta>0, \quad q_{i} \geq 0, \quad i=0,1, \ldots, r-1 ;
\end{gathered}
$$

(C3) $\sum_{s=k_{0}}^{k-2}\left\|g_{k, s}\left(x_{0}, x_{1}, \ldots, x_{r}\right)\right\| \leq M<\infty, \quad M \in R^{+}$.
Denote by

$$
P=\sum_{r=0}^{r} p_{i}, \quad Q=\sum_{i=1}^{r} q_{i}
$$

Using the method in [10], we can obtain the following theorem.

Theorem 4. Under the hypothesises (C1, C2, and C3), system (2) is asymptotically stable if $P>1, \alpha>0,0<\beta<1$, and $M<1 / 2 \alpha$.

Proof. Since $P>1$, we can choose two real numbers $s \geq 1$, $0<\delta<1$ such that $P-s>0$,

$$
\begin{gathered}
p_{0}+\frac{p_{1}}{s}+\cdots+\frac{p_{r}}{s^{r}}-s>0, \\
\alpha \beta \delta^{p_{0}+p_{1} / s+\cdots+p_{r} / s^{r}-s}<\frac{1}{2} .
\end{gathered}
$$

From (7), it is easy to note that

$$
\begin{gathered}
p_{0}+\frac{p_{1}+q_{1}}{s}+\cdots+\frac{p_{r}+q_{r}}{s^{r}}-s>0, \\
\alpha \beta \delta^{p_{0}+\left(p_{1}+q_{1}\right) / s+\cdots+\left(p_{r}+q_{r}\right) / s^{r}-s}<\frac{1}{2} .
\end{gathered}
$$

Next, we consider solution of the system (2) with the initial condition such that $\left\|y_{k}\right\| \leq \delta, k=0,-1, \ldots,-r$. According to the definition, we will prove the following inequality:

$$
\left\|y_{k}\right\| \leq \delta^{s^{k}}, \quad \forall k \in Z^{+}
$$

which is obvious to hold for $k=1$.

Assuming that (9) still holds for $2,3, \ldots, k(k \geq 2)$, in addition to $(C 2)$ and $(C 3)$, then we estimate the solution at the $(k+1)$ step by $(4)$.

$$
\text { Let } z_{r+1}=\sum_{s=0}^{k-1} g_{k, s}\left(y_{s}, y_{s-1}, \ldots, y_{s-r+1}\right) \text {. Then }
$$

$$
\begin{aligned}
\left\|z_{r+1}\right\|= & \left\|\sum_{s=0}^{k-1} g_{k, s}\left(y_{s}, y_{s-1}, \ldots, y_{s-r+1}\right)\right\| \\
= & \| g_{k, k-1}\left(y_{k-1}, y_{k-2}, \ldots, y_{k-r}\right) \\
& +\sum_{s=0}^{k-2} g_{k, s}\left(y_{s}, y_{s-1}, \ldots, y_{s-r+1}\right) \| \\
\leq & \left\|\beta y_{k-1}^{q_{1}} y_{k-2}^{q_{2}} \ldots y_{k-r}^{q_{r}}\right\|+M \\
\leq & \beta \delta^{s^{k-1} q_{1}+s^{k-2} q_{2}+\cdots+s^{k-r}} q_{r}+M .
\end{aligned}
$$


By the inequalities (8) and (10), we can obtain the following conclusion:

$$
\begin{aligned}
\left\|y_{k+1}\right\|= & \left\|f_{k}\left(y_{0}, y_{1}, \ldots, z_{r+1}\right)\right\| \\
\leq & \alpha \prod_{i=0}^{r}\left\|y_{i}\right\|^{p_{i}}\left\|z_{r+1}\right\| \\
\leq & \alpha\left\|y_{k}\right\|^{p_{0}}\left\|y_{k-1}\right\|^{p_{1}} \cdots\left\|y_{k-r}\right\|^{p_{r}} \\
& \times\left(\beta \delta^{s^{k-1} q_{1}+s^{k-2} q_{2}+\cdots+s^{k-r} q_{r}}+M\right) \\
\leq & \alpha \delta^{s^{k} p_{0}+s^{k-1} p_{1}+\cdots+s^{k-r} p_{r}}\left(\beta \delta^{s^{k-1}} q_{1}+s^{k-2} q_{2}+\cdots+s^{k-r} q_{r}+M\right) \\
= & \alpha \beta \delta^{s^{k} p_{0}+s^{k-1}\left(p_{1}+q_{1}\right)+\cdots+s^{k-r}\left(p_{r}+q_{r}\right)} \\
& +\alpha \delta^{s^{k} p_{0}+s^{k-1} p_{1}+\cdots+s^{k-r} p_{r}} M \\
= & \delta^{s^{k+1}}\left(\alpha \beta \delta^{s^{k}\left(p_{0}+\left(p_{1}+q_{1}\right) / s+\cdots+\left(p_{r}+q_{r}\right) / s^{r}-s\right)}\right. \\
& \left.+\alpha \delta^{s^{k}\left(p_{0}+p_{1} / s+\cdots+p_{r} / s^{r}-s\right)} M\right) \leq \delta^{s^{k+1}},
\end{aligned}
$$

which implies that inequality (9) holds for the step $(k+1)$. Therefore, inequality (9) holds for all $k \in Z^{+}$. Since $\delta \in(0,1)$, we can obtain that $s^{k} \rightarrow \infty$ as $k \rightarrow \infty$ and derive the conclusion. Hence, the proof is completed.

Theorem 5. Let system (2) satisfy the same conditions as in Theorem 4 . Then, the zero solutions are asymptotically stable if $P=1,0<\alpha \beta<1$, and $M<\beta\left(1-\delta^{\mathrm{Q}}\right)$.

Proof. According to the assumption, we can define a set for every integer $n \geq 0$,

$$
I_{n}=\{n(r+1)+i: i=1,2, \ldots, r+1\},
$$

which contains $r+1$ index numbers and

$$
Z^{+}=\bigcup_{n=0}^{\infty} I_{n}
$$

To prove that the solution is asymptotically stable, according to the definition, we will prove the following inequality:

$$
\left\|y_{k}\right\| \leq(\alpha \beta)^{n+1} \delta, \quad \forall k \in I_{n} .
$$

It is obvious that (9) holds for $k=1$. Since the definition of the set $I_{n}$ and the assumption of the solution $\left\{y_{k}\right\}$ fit the estimation (14) at the step $k$, the following proof will be divided into two aspects. (i) If $k$ is on the end of $I_{n}$, such that $k+1 \in I_{n+1}$, then we have

$$
\begin{aligned}
& \left\|y_{k+1}\right\| \\
& \leq \alpha \beta\left(\left((\alpha \beta)^{n+1} \delta\right)^{p_{0}}\left((\alpha \beta)^{n+1} \delta\right)^{p_{1}+q_{1}} \cdots\left((\alpha \beta)^{n+1} \delta\right)^{p_{r}+q_{r}}\right) \\
& \quad+\alpha(\alpha \beta)^{n+1} \delta^{p_{0}}\left((\alpha \beta)^{n+1} \delta\right)^{p_{1}} \cdots\left((\alpha \beta)^{n+1} \delta\right)^{p_{r}} M \\
& =\alpha \beta\left(\left((\alpha \beta)^{n+1} \delta\right)^{P+Q}+\alpha(\alpha \beta)^{n+1} \delta\right)^{P} M .
\end{aligned}
$$

By the assumptions in this theorem, we have

$$
\left\|y_{k+1}\right\| \leq(\alpha \beta)^{n+2} \delta\left(\delta^{Q}+\beta^{-1} M\right) \leq(\alpha \beta)^{n+2} .
$$

As desired.

(ii) If $k$ is inside $I_{n}$, in other words, $k, k-1, \ldots, k-i \in I_{n}$ and $k-i-1, k-i-2, \ldots, k-r \in I_{n-1}$, such that $k+1 \in I_{n+1}$, then we have

$$
\begin{aligned}
\left\|y_{k+1}\right\| \leq & \alpha \beta\left(\left((\alpha \beta)^{n+1} \delta\right)^{p_{0}} \cdots\left((\alpha \beta)^{n+1} \delta\right)^{p_{i}+q_{i}}\right. \\
& \left.\times\left((\alpha \beta)^{n} \delta\right)^{p_{i+1}+q_{i+1}} \cdots\left((\alpha \beta)^{n} \delta\right)^{p_{r}+q_{r}}\right) \\
+\alpha & \left((\alpha \beta)^{n+1} \delta\right)^{p_{0}} \cdots\left((\alpha \beta)^{n+1} \delta\right)^{p_{i}} \\
& \left.\times\left((\alpha \beta)^{n} \delta\right)^{p_{i+1}} \cdots\left((\alpha \beta)^{n} \delta\right)^{p_{r}}\right) M .
\end{aligned}
$$
derive

Because of the sufficient condition in this theorem, we can

$$
\left\|y_{k+1}\right\| \leq \alpha \beta\left((\alpha \beta)^{n} \delta\right)^{P+Q}+\alpha\left((\alpha \beta)^{n} \delta\right)^{P} M \leq(\alpha \beta)^{n+1} \delta,
$$

which proves that (14) holds for $y_{k+1}$. From estimation (14) and $\alpha \beta \in(0,1)$, it follows the asymptotic stability of the zero solutions of system (2). The proof is ended.

\section{Instability Conditions}

Apart from the instability comparison condition defined in the whole state space $Y=R^{n}$ in [13], we state that the condition is satisfied only in some subset of the state space $Y$. Thus, we introduce a convex set $G \subset Y, 0 \in G$ to be an invariant set for system (2). Further, for every $k_{0} \in Z^{+}$, the solution $y_{k}$ with the initial condition (3) starting in $G$ : $y_{n} \in G$, $n=0,-1, \ldots,-r$ satisfies $y_{k} \in G$ for all $k \in Z^{+}$.

In this section, we assume the following comparison condition:

$$
\begin{array}{r}
\left\|f_{k}\left(x_{0}, x_{1}, \ldots, x_{r+1}\right)\right\| \geq \widetilde{\alpha} \prod_{i=0}^{r}\left\|x_{i}\right\|^{\tilde{p}_{i}}\left\|x_{r+1}\right\|, \\
\tilde{\alpha}>0, \quad \tilde{p}_{i} \geq 0
\end{array}
$$


(C5) $\left\|g_{k, s}\left(x_{0}, x_{1}, \ldots, x_{r-1}\right)\right\| \geq \widetilde{\beta} \prod_{i=1}^{r}\left\|x_{i-1}\right\|^{\widetilde{q}_{i}}$

$$
\widetilde{\beta}>0, \quad \tilde{q}_{i} \geq 0 .
$$

Denote by

$$
\widetilde{P}=\sum_{r=0}^{r} \widetilde{p}_{i}, \quad \widetilde{Q}=\sum_{i=0}^{r-1} \widetilde{q}_{i}
$$

The following three theorems are our main results on instability of the system (2).

Theorem 6. Under conditions (C4)-(C5), system (2) is unstable if $0<\widetilde{P}+\widetilde{Q}<1$ for all $0<\widetilde{\alpha} \widetilde{\beta}<1$.

Proof. Let the arbitrary initial conditions satisfy

$$
\begin{gathered}
\left\|y_{i}\right\|=\eta_{i}, \\
i=0,-1, \ldots,-r, \quad \eta=\min \left\{1, \eta_{i}: i=0,-1,-2, \ldots,-r\right\} .
\end{gathered}
$$

Note that $\left\|y_{i}\right\| \geq \eta$, for all $i=0,-1, \ldots,-r$.

For $k=1$, from (2) and conditions (C4), (C5), we have

$$
\begin{aligned}
y_{1} & =f_{0}\left(y_{0}, y_{-1}, \ldots, y_{-r}\right) \geq \widetilde{\alpha} \prod_{i=0}^{r}\left\|y_{i}\right\|^{\widetilde{p}_{i}}=\widetilde{\alpha} \prod_{i=0}^{r} \eta_{i}^{\widetilde{p}_{i}} \\
& \geq \widetilde{\alpha} \eta^{\widetilde{p}_{0}+\widetilde{p}_{1}+\cdots+\widetilde{p}_{r}}=\widetilde{\alpha} \eta \widetilde{P} \geq \widetilde{\alpha} \widetilde{\beta} \eta^{\widetilde{P}} .
\end{aligned}
$$

For $k=2$, by the same way and noting that $G$ is invariant set, we have the following estimation:

$$
\begin{aligned}
& y_{2}=f_{1}\left(y_{1}, y_{0}, \ldots, y_{-r+1}, g_{1,0}\left(y_{0}, \ldots, y_{-r+1}\right)\right) \\
& \geq \widetilde{\alpha}\left(\widetilde{\alpha} \widetilde{\beta} \eta^{\widetilde{P}}\right)^{\tilde{p}_{0}} y_{0} \tilde{p}_{1} y_{-1} \tilde{p}_{2} \cdots y_{-r+1} \tilde{p}_{r} \widetilde{\beta} y_{0} \widetilde{q}_{1} y_{-1} \tilde{q}_{2} \cdots y_{-r+1} \tilde{q}_{r} \\
& \geq \widetilde{\alpha}\left(\widetilde{\alpha} \widetilde{\beta} \eta^{\widetilde{P}}\right)^{\tilde{p}_{0}} \eta^{\tilde{p}_{1}} \eta^{\tilde{p}_{2}} \cdots \eta^{\tilde{p}_{r}} \widetilde{\beta} \eta^{\widetilde{q}_{1}} \eta^{\tilde{q}_{2}} \cdots \eta^{\tilde{q}_{r}} \\
& =(\widetilde{\alpha} \widetilde{\beta})^{1+\widetilde{p}_{0}} \eta^{\widetilde{P} \widetilde{p}_{0}+\widetilde{p}_{1}+\cdots+\widetilde{p}_{r}+\widetilde{q}_{1}+\widetilde{q}_{2}+\cdots+\widetilde{q}_{r}} .
\end{aligned}
$$

Repeating the above procedure, for every step $k \in Z^{+}$, we can obtain the inequality of the form

$$
\|y(k)\| \geq(\widetilde{\alpha} \widetilde{\beta})^{\mu(k)} \eta^{\nu(k)},
$$

where the functions $\mu(k), v(k)$ are defined by the following two propositions. (a) The functions $\mu(k), \nu(k)$ satisfy the following relations:

$$
\begin{gathered}
\mu(0)=\mu(-1)=\cdots=\mu(-r)=0, \\
\mu(k+1)=1+\widetilde{p}_{0} \mu(k)+\left(\widetilde{p}_{1}+\widetilde{q}_{1}\right) \mu(k-1) \\
+\cdots+\left(\widetilde{p}_{r}+\widetilde{q}_{r}\right) \mu(k-r), \\
\nu(0)=\nu(-1)=\cdots=\nu(-r)=1, \\
\nu(k+1)=\tilde{p}_{0} \nu(k)+\left(\tilde{p}_{1}+\widetilde{q}_{1}\right) \nu(k-1) \\
+\cdots+\left(\widetilde{p}_{r}+\widetilde{q}_{r}\right) \nu(k-r),
\end{gathered}
$$

which can be directly proved by the method of induction in $k \in Z^{+}$.

(b) The functions $\mu(k), \nu(k)$ satisfy the following estimations:

$$
\sum_{i=0}^{n}(\widetilde{P}+\widetilde{Q})^{i} \leq \mu(k) \leq \sum_{i=0}^{k-1}(\widetilde{P}+\widetilde{Q})^{i}, \quad \forall k \in I_{n}, n \in Z^{+} ;
$$

$\nu(k) \leq(\widetilde{P}+\widetilde{Q})^{n}, \quad$ if $(\widetilde{P}+\widetilde{Q}) \in(0,1], \forall k \in I_{n}, n \in Z^{+}$,

where $I_{n}$ is defined by (12).

The proof of Proposition (b) is as follows. We will prove the left-hand side inequality (27) by induction on each set $I_{n}$, and the right-hand side can be proved similarly.

It is obvious that the assertion is true for the step $k=1$. Assume that the assertion holds until the step $k \in I$. For every $k \in I_{n}$, using Proposition (a), (24) and by induction assumption, we have

$$
\begin{aligned}
\mu(k+1)= & 1+\widetilde{p}_{0} \mu(k)+\left(\widetilde{p}_{1}+\widetilde{q}_{1}\right) \mu(k-1) \\
& +\cdots+\left(\widetilde{p}_{r}+\widetilde{q}_{r}\right) \mu(k-r) .
\end{aligned}
$$

If $k \in I$ is on the end of the set $I_{n}$, that is, $k \in I_{n},(k+1) \in I_{n+1}$, we have

$$
\begin{aligned}
\mu(k+1) \geq & 1+\widetilde{p}_{0}\left[1+(\widetilde{P}+\widetilde{Q})+\cdots+(\widetilde{P}+\widetilde{Q})^{n}\right] \\
& +\left(\widetilde{p}_{1}+\widetilde{q}_{1}\right)\left[1+(\widetilde{P}+\widetilde{Q})+\cdots+(\widetilde{P}+\widetilde{Q})^{n}\right] \\
& +\cdots+\left(\widetilde{p}_{r}+\widetilde{q}_{r}\right)\left[1+(\widetilde{P}+\widetilde{Q})+\cdots+(\widetilde{P}+\widetilde{Q})^{n}\right] \\
= & 1+\left[1+(\widetilde{P}+\widetilde{Q})+\cdots+(\widetilde{P}+\widetilde{Q})^{n}\right] \\
& \times\left[\widetilde{p}_{0}+\left(\widetilde{p}_{1}+\widetilde{q}_{1}\right)+\cdots+\left(\widetilde{p}_{r}+\widetilde{q}_{r}\right)\right] \\
= & 1+(\widetilde{P}+\widetilde{Q})+\cdots+(\widetilde{P}+\widetilde{Q})^{n+1} .
\end{aligned}
$$


If $k$ is inside $I_{n}$, so that $k, k-1, \ldots, k-i \in I_{n}$ and $k-i-1$, $k-i-2, \ldots, k-r \in I_{n-1}$, which implies $k+1 \in I_{n+1}$, then we have

$$
\begin{aligned}
& \mu(k+1) \geq 1+\widetilde{p}_{0}\left[1+(\widetilde{P}+\widetilde{Q})+\cdots+(\widetilde{P}+\widetilde{Q})^{n}\right] \\
& +\left(\widetilde{p}_{1}+\widetilde{q}_{1}\right)\left[1+(\widetilde{P}+\widetilde{Q})+\cdots+(\widetilde{P}+\widetilde{Q})^{n}\right] \\
& +\cdots+\left(\widetilde{p}_{i}+\widetilde{q}_{i}\right)\left[1+(\widetilde{P}+\widetilde{Q})+\cdots+(\widetilde{P}+\widetilde{Q})^{n}\right] \\
& +\left(\widetilde{p}_{i+1}+\widetilde{q}_{i+1}\right)\left[1+(\widetilde{P}+\widetilde{Q})+\cdots+(\widetilde{P}+\widetilde{Q})^{n}\right] \\
& +\cdots+\left(\widetilde{p}_{r}+\widetilde{q}_{r}\right)[1+(\widetilde{P}+\widetilde{Q})] \\
& =1+\left[\widetilde{p}_{0}+\left(\widetilde{p}_{1}+\tilde{q}_{1}\right)+\cdots+\left(\widetilde{p}_{r}+\tilde{q}_{r}\right)\right]+(\widetilde{P}+\widetilde{Q}) \\
& \times\left[\tilde{p}_{0}+\left(\tilde{p}_{1}+\tilde{q}_{1}\right)+\cdots+\left(\tilde{p}_{r}+\tilde{q}_{r}\right)\right]+\cdots \\
& +(\widetilde{P}+\widetilde{Q})^{n-1}\left[\widetilde{p}_{0}+\left(\widetilde{p}_{1}+\widetilde{q}_{1}\right)+\cdots+\left(\widetilde{p}_{r}+\widetilde{q}_{r}\right)\right] \\
& +(\widetilde{P}+\widetilde{Q})^{n}\left[\widetilde{p}_{0}+\left(\widetilde{p}_{1}+\widetilde{q}_{1}\right)+\cdots+\left(\widetilde{p}_{i}+\widetilde{q}_{i}\right)\right] \\
& \geq 1+\left[\tilde{p}_{0}+\left(\tilde{p}_{1}+\tilde{q}_{1}\right)+\cdots+\left(\tilde{p}_{r}+\tilde{q}_{r}\right)\right] \\
& +(\widetilde{P}+\widetilde{Q})\left[\tilde{p}_{0}+\left(\tilde{p}_{1}+\widetilde{q}_{1}\right)+\cdots+\left(\tilde{p}_{r}+\widetilde{q}_{r}\right)\right]+\cdots \\
& +(\widetilde{P}+\widetilde{Q})^{n-1}\left[\widetilde{p}_{0}+\left(\widetilde{p}_{1}+\widetilde{q}_{1}\right)+\cdots+\left(\widetilde{p}_{r}+\widetilde{q}_{r}\right)\right] \\
& =1+(\widetilde{P}+\widetilde{Q})+\cdots+(\widetilde{P}+\widetilde{Q})^{n},
\end{aligned}
$$

which proves the left-hand side inequality of (27).

To prove inequality (28), using (26) and by induction, we assume that the inequality holds for all steps $1,2, \ldots, k$. For the step $k+1$, by the same method of induction, if $k \in I$ and is on the end of the set $I$ so that $k+1 \in I_{n+1}$, we have

$$
\begin{aligned}
v(k+1)= & \widetilde{p}_{0} v(k)+\left(\widetilde{p}_{1}+\widetilde{q}_{1}\right) v(k-1)+\cdots \\
& +\left(\widetilde{p}_{r}+\widetilde{q}_{r}\right) v(k-r) \\
\leq & \widetilde{p}_{0}(\widetilde{P}+\widetilde{Q})^{n}+\left(\widetilde{p}_{1}+\widetilde{q}_{1}\right)(\widetilde{P}+\widetilde{Q})^{n}+\cdots \\
& +\left(\widetilde{p}_{r}+\widetilde{q}_{r}\right)(\widetilde{P}+\widetilde{Q})^{n}=(\widetilde{P}+\widetilde{Q})^{n} .
\end{aligned}
$$

If $k$ is inside $I_{n}$, in other words, $k, k-1, \ldots, k-i \in I_{n}$ and $k-i-1, k-i-2, \ldots, k-r \in I_{n-1}$, such that $k+1 \in I_{n+1}$, then we have

$$
\begin{aligned}
v(k+1) \leq & \widetilde{p}_{0}(\widetilde{P}+\widetilde{Q})^{n}+\left(\widetilde{p}_{1}+\widetilde{q}_{1}\right)(\widetilde{P}+\widetilde{Q})^{n}+\cdots \\
& +\left(\widetilde{p}_{i}+\widetilde{q}_{i}\right)(\widetilde{P}+\widetilde{Q})^{n}+\left(\widetilde{p}_{i+1}+\widetilde{q}_{i+1}\right)(\widetilde{P}+\widetilde{Q})^{n-1} \\
& +\cdots+\left(\widetilde{p}_{r}+\widetilde{q}_{r}\right)(\widetilde{P}+\widetilde{Q})^{n-1} .
\end{aligned}
$$

Given $(\widetilde{P}+\widetilde{Q}) \in(0,1):(\widetilde{P}+\widetilde{Q})^{n-1} \geq(\widetilde{P}+\widetilde{Q})^{n}$, we obtain

$$
\begin{aligned}
v(k+1) \leq & \widetilde{p}_{0}(\widetilde{P}+\widetilde{Q})^{n-1}+\left(\widetilde{p}_{1}+\widetilde{q}_{1}\right)(\widetilde{P}+\widetilde{Q})^{n-1} \\
& +\cdots+\left(\widetilde{p}_{i}+\widetilde{q}_{i}\right)(\widetilde{P}+\widetilde{Q})^{n-1}+\left(\widetilde{p}_{i+1}+\widetilde{q}_{i+1}\right) \\
& \times(\widetilde{P}+\widetilde{Q})^{n-1}+\cdots+\left(\widetilde{p}_{r}+\widetilde{q}_{r}\right)(\widetilde{P}+\widetilde{Q})^{n-1} \\
= & {\left[\widetilde{p}_{0}+\left(\widetilde{p}_{1}+\widetilde{q}_{1}\right)+\cdots+\left(\widetilde{p}_{r}+\widetilde{q}_{r}\right)\right](\widetilde{P}+\widetilde{Q})^{n-1} } \\
= & (\widetilde{P}+\widetilde{Q})^{n}
\end{aligned}
$$

as desired.

Next, we will prove Theorem 6 based on the above propositions. Let $\widetilde{P}+\widetilde{Q} \in(0,1)$. From the estimation (24) and the estimations (27), (28) of Proposition (b), we have two cases.

Case (i). If $(\widetilde{\alpha} \widetilde{\beta}) \geq 1$, we have

$$
\begin{array}{r}
(\widetilde{\alpha} \widetilde{\beta})^{\mu(k)} \geq(\widetilde{\alpha} \widetilde{\beta})^{1+(\widetilde{P}+\widetilde{Q})+\cdots+(\widetilde{P}+\widetilde{Q})^{n}}, \quad \eta^{\nu(k)} \geq \eta^{(\widetilde{P}+\widetilde{Q})^{n},} \\
\forall k \in I_{n}, \quad n=1,2, \ldots,
\end{array}
$$

where $\eta \geq 1$. Therefore,

$$
\begin{aligned}
\left\|y_{k}\right\| & \geq(\widetilde{\alpha} \widetilde{\beta})^{1+(\widetilde{P}+\widetilde{Q})+\cdots+(\widetilde{P}+\widetilde{Q})^{n}} \eta^{(\widetilde{P}+\widetilde{Q})^{n}} \\
& =(\widetilde{\alpha} \widetilde{\beta})^{1 /[1-(\widetilde{P}+\widetilde{Q})]}(\widetilde{\alpha} \widetilde{\beta})^{(\widetilde{P}+\widetilde{Q})^{n+1} /[(\widetilde{P}+\widetilde{Q})-1]} \eta^{(\widetilde{P}+\widetilde{Q})^{n},}
\end{aligned}
$$

which gives

$$
\lim \left\|y_{k}\right\| \geq(\widetilde{\alpha} \widetilde{\beta})^{1 /[1-(\widetilde{P}+\widetilde{Q})]},
$$

as $n$ and then $k$ go to infinity.

Therefore, there are a number $a>0$ and a large number $K \in Z^{+}$such that $\left\|y_{K}\right\| \geq a$. By Definition 3 , if $\varepsilon=a$ and $\delta \geq \eta$, the zero solutions are unstable.

Case (ii). If $(\widetilde{\alpha} \widetilde{\beta}) \in(0,1)$, using the estimation (24) and the estimations (27), (28) of Proposition (b) again, we then obtain

$$
\begin{array}{r}
(\widetilde{\alpha} \widetilde{\beta})^{\mu(k)} \geq(\widetilde{\alpha} \widetilde{\beta})^{1+(\widetilde{P}+\widetilde{Q})+\cdots+(\widetilde{P}+\widetilde{Q})^{k-1}}, \quad \eta^{\nu(k)} \geq \eta^{(\widetilde{P}+\widetilde{Q})^{n},} \\
\forall k \in I_{n}, \quad n=1,2, \ldots,
\end{array}
$$

where $\eta \geq 1$. Therefore,

$$
\begin{aligned}
\left\|y_{k}\right\| & \geq(\widetilde{\alpha} \widetilde{\beta})^{1+(\widetilde{P}+\widetilde{Q})+\cdots+(\widetilde{P}+\widetilde{Q})^{k-1}} \eta^{(\widetilde{P}+\widetilde{Q})^{n}} \\
& =(\widetilde{\alpha} \widetilde{\beta})^{1 /[1-(\widetilde{P}+\widetilde{Q})]}(\widetilde{\alpha} \widetilde{\beta})^{(\widetilde{P}+\widetilde{Q})^{k} /[(\widetilde{P}+\widetilde{Q})-1]} \eta^{(\widetilde{P}+\widetilde{Q})^{n},}
\end{aligned}
$$

which gives

$$
\lim \left\|y_{k}\right\| \geq(\widetilde{\alpha} \widetilde{\beta})^{1 /[1-(\widetilde{P}+\widetilde{Q})]},
$$

as $n$ and then $k$ goes to infinity. Thus, as in Case (i), we prove the instability of zero solutions. The proof is ended. 
According to Theorem 6, we can analogize the following theorems.

Theorem 7. Assume that conditions (C4)-(C5), where we estimate inequality (24), hold in the system (2). Then, system (2) is unstable if $\widetilde{P}+\widetilde{Q}=1$ for all $(\widetilde{\alpha} \widetilde{\beta})>1$.

Proof. Let $\widetilde{P}+\widetilde{Q}=1$ and $(\widetilde{\alpha} \widetilde{\beta})>1$. In this case for the inequality we obtained in Theorem 6

$$
\left\|y_{k}\right\| \geq(\widetilde{\alpha} \widetilde{\beta})^{1 /[1-(\widetilde{P}+\widetilde{Q})]}(\widetilde{\alpha} \widetilde{\beta})^{(\widetilde{P}+\widetilde{Q})^{n+1} /[(\widetilde{P}+\widetilde{Q})-1]} \eta^{(\widetilde{P}+\widetilde{Q})^{n}},
$$

we have

$$
\left\|y_{k}\right\| \geq(\widetilde{\alpha} \widetilde{\beta})^{n+1} \eta, \quad k \in I_{n}
$$

which goes to infinity as $n, k$ go to infinity.

By Definition 3 of the instability, it is easy to see that the zero solutions are unstable. The theorem is proved.

Theorem 8. Assume that conditions (C4)-(C5), where we estimate inequality (24), hold in the system (2). Then, system (2) is asymptotically unstable if $\widetilde{P}+\widetilde{Q}=\widetilde{\alpha} \widetilde{\beta}=1$.

Proof. When $\widetilde{P}+\widetilde{Q}=\widetilde{\alpha} \widetilde{\beta}=1$, in addition to the inequality

$$
\left\|y_{k}\right\| \geq(\widetilde{\alpha} \widetilde{\beta})^{n+1} \eta
$$

we can obtain

$$
\left\|y_{k}\right\| \geq \eta, \quad \forall k \in Z^{+}
$$

which shows that the zero solutions are asymptotically unstable. This ends the proof.

\section{Applications}

In this section, we state two examples concerning the above theorems in Sections 2 and 3, respectively.

Example 9. The difference control system with higher order term

$$
y_{k+1}=f_{k}\left(y_{k}, y_{k-1}, \varphi_{k-2}, \sum_{s=0}^{k-1} g_{k, s}\left(y_{s}, y_{s-1}\right)\right), \quad k \in Z^{+} \text {, }
$$

where $f_{k}(\cdot): Z^{+} \times Y \times Y \times T \times Y, \varphi_{k} \in T$, is a control argument. We note that the system is stabilizable when there is a linear bounded operator $\Phi: Y \rightarrow T$ such that the control $\varphi_{k}=$ $\Phi\left(y_{k}\right)$, if the system without control

$$
y_{k+1}=f_{k}\left(y_{k}, y_{k-1}, \Phi y_{k-2}, \sum_{s=0}^{k-1} g_{k, s}\left(y_{s}, y_{s-1}\right)\right)
$$

is asymptotically stable.
Suppose that the function $f_{k}(\cdot)$ satisfies the conditions

$$
\begin{array}{r}
\sum_{s=0}^{k-1} g_{k, s}\left(y_{s}, y_{s-1}\right) \leq z\left(y_{k}, y_{k-1}, y_{k-2}\right), \\
\left\|f_{k}(\cdot)\right\| \leq \lambda\|x\|^{p_{1}}\|y\|^{p_{2}}\|t\|^{p_{3}}, \\
\forall k \in Z^{+}, \quad(x, y, t, z) \in X \times X \times T \times X,
\end{array}
$$

for some $p_{1}, p_{2}, p_{3} \in Z^{+}$. Then by Theorems 6 and 7 , the above system is stable under the following control $\varphi_{k}=\Phi\left(y_{k}\right)$ and one of the following conditions:

$$
p_{1}+p_{2}+p_{3}>1
$$

or

$$
p_{1}+p_{2}+p_{3}=1, \quad\|\Phi\|<\frac{1}{\lambda} .
$$

Example 10. We study the following system in Banach space $l^{2}:$

$$
y_{k+1}=f_{k}\left(y_{k}, y_{k-1}, y_{k-2}, \sum_{s=0}^{k-1} g_{k, s}\left(y_{s}, y_{s-1}\right)\right), \quad k \in Z^{+},
$$

where

$$
\begin{aligned}
f_{k}(\cdot)= & \left(a_{0}\left\|y_{k}\right\|^{q_{0}}, a_{1}\left\|y_{k-1}\right\|^{q_{1}}, a_{2}\left\|y_{k-2}\right\|^{q_{2}},\right. \\
& \left.\sqrt{a_{3}\left(\left\|y_{k}+y_{k-1}+y_{k-2}\right\|^{2 q_{3}}\right)}, 0,0, \ldots\right)
\end{aligned}
$$

and $a_{i}>0, q_{i}>0, i=0,1,2,3$, are real numbers. We assume that

$$
\left\|y_{k}+y_{k-1}+y_{k-2}\right\| \geq\left\|y_{k-1}\right\|+\left\|y_{k}-2\right\| .
$$

Next, we can take a convex cone $G$ with the form of

$$
\begin{gathered}
G=\left\{\left(y_{0}, y_{1}, y_{2}, y_{3}, \ldots\right) \in l^{2}: y_{i} \geq 0,\right. \\
\left.i=0,1,2,3 ; y_{j}=0, \forall j \geq 0\right\}
\end{gathered}
$$

which is the invariant set for system.

By Theorems 6 and 7 and for arbitrary $\left(y_{k}, y_{k-1}, y_{k-2}\right.$, $\left.\sum_{s=0}^{k-1} g_{k, s}\left(y_{s}, y_{s-1}\right)\right) \in G$, we have

$$
\begin{aligned}
\left\|f_{k}(\cdot)\right\|^{2}= & \|\left(a_{0}\left\|y_{k}\right\|^{q_{0}}, a_{1}\left\|y_{k-1}\right\|^{q_{1}}, a_{2}\|y(k-2)\|^{q_{2}},\right. \\
& \left.\sqrt{a_{3}\left(\left\|y_{k}+y_{k-1}+y_{k-2}\right\|^{2 q_{3}}\right)}, 0,0, \ldots\right) \|^{2} \\
\geq & \sum_{i=0}^{3} a_{i}^{2}\left\|y_{k-i}\right\|^{2 p_{i}},
\end{aligned}
$$

where $p_{0}=q_{0}, p_{1}=q_{1}+q_{3}$, and $p_{2}=q_{2}+q_{3}$. Thus,

$$
\left\|f_{k}(\cdot)\right\| \geq \sqrt{3}\left(a_{0} a_{1} a_{2}\right)^{1 / 3}\left\|y_{k}\right\|^{p_{0} / 3}\left\|y_{k-1}\right\|^{p_{1} / 3}\left\|y_{k-2}\right\|^{p_{2} / 3} .
$$

Hence, system (50) is unstable if one of the following conditions holds:
(a') $p_{0}+p_{1}+p_{2}<3$
(b') $p_{0}+p_{1}+p_{2}=3$ and $a_{0} a_{1} a_{2}>1 / \sqrt{27}$. 


\section{Conflict of Interests}

The authors declare that there is no conflict of interests regarding the publication of this paper.

\section{Acknowledgment}

The project was supported by the National Natural Science Foundation of China 11271261, 11171178, and FP7-PEOPLE2012-IRSES-316338.

\section{References}

[1] R. P. Agarwal, Difference Equations and Inequalities. Theory, Methods, and Applications, vol. 228 of Monographs and Textbooks in Pure and Applied Mathematics, Marcel Dekker, New York, NY, USA, 2nd edition, 2000.

[2] S. Elaydi, An Introduction to Difference Equations, Springer, New York, NY, USA, 1995.

[3] V. L. Kocić and G. Ladas, Global Behavior of Nonlinear Difference Equations of Higher Order with Applications, vol. 256 of Mathematics and Its Applications, Kluwer Academic Publishers Group, Dordrecht, The Netherlands, 1993.

[4] A. Y. T. Leung, J. N. Xu, and W. S. Tsui, "Nonlinear delay difference equations for housing dynamics assuming heterogeneous backward-looking expectations," Applied Mathematics and Mechanics, vol. 28, no. 6, pp. 785-798, 2007.

[5] P. Cull, M. Flahive, and R. Robson, Difference Equations. From Rabbits to Chaos, Springer, New York, NY, USA, 2005.

[6] V. N. Phat, Constrained Control Problems of Discrete Processes, World Scientific, Singapore, 1996.

[7] M. M. Kipnis and D. A. Komissarova, "A note on explicit stability conditions for autonomous higher order difference equations," Journal of Difference Equations and Applications, vol. 13, no. 5, pp. 457-461, 2007.

[8] S. Mohamad and K. Gopalsamy, "Exponential stability of continuous-time and discrete-time cellular neural networks with delays," Applied Mathematics and Computation, vol. 135, no. 1, pp. 17-38, 2003.

[9] J. Yao and F. Meng, "Asymptotic behavior of solutions of certain higher order nonlinear difference equation," Journal of Computational and Applied Mathematics, vol. 205, no. 1, pp. 640-650, 2007.

[10] N. S. Bay and V. N. Phat, "Stability analysis of nonlinear retarded difference equations in Banach spaces," Computers \& Mathematics with Applications, vol. 45, no. 6-9, pp. 951-960, 2003.

[11] J. P. LaSalle, The Stability and Control of Discrete Processes, vol. 62 of Applied Mathematical Sciences, Springer, New York, NY, USA, 1986.

[12] R. Naulin and M. Pinto, "Stability of discrete dichotomies for linear difference systems," Journal of Difference Equations and Applications, vol. 3, no. 2, pp. 101-123, 1997.

[13] R. Naulin and C. Vanegas, "Instability of discrete-time systems," Electronic Journal of Differential Equations, vol. 33, pp. 1-11, 1998.

[14] H. M. Xiao and Y. Q. Liu, "The stability of linear time-varying discrete systems with time-delay," Journal of Mathematical Analysis and Applications, vol. 188, no. 1, pp. 66-77, 1994.

[15] X. S. Yang and Y. Stepanenko, "A stability criterion for discrete nonlinear systems with time delayed feedback," IEEE Transactions on Automatic Control, vol. 39, no. 3, pp. 585-589, 1994. 


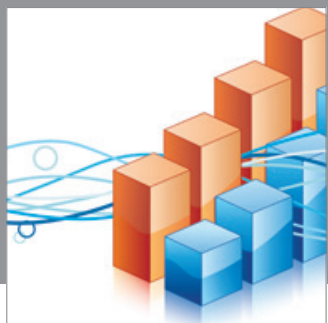

Advances in

Operations Research

mansans

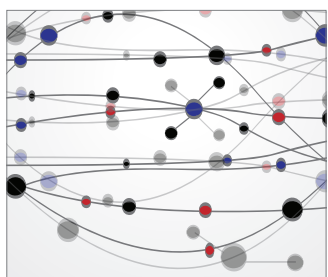

The Scientific World Journal
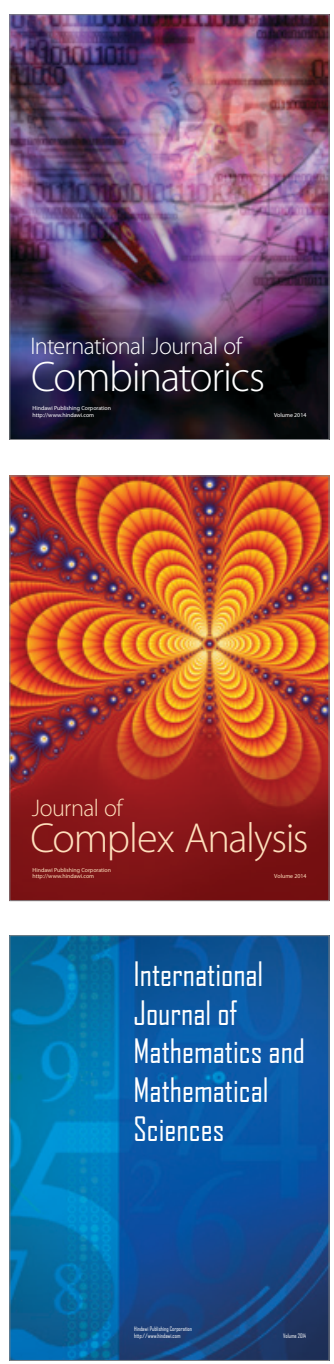
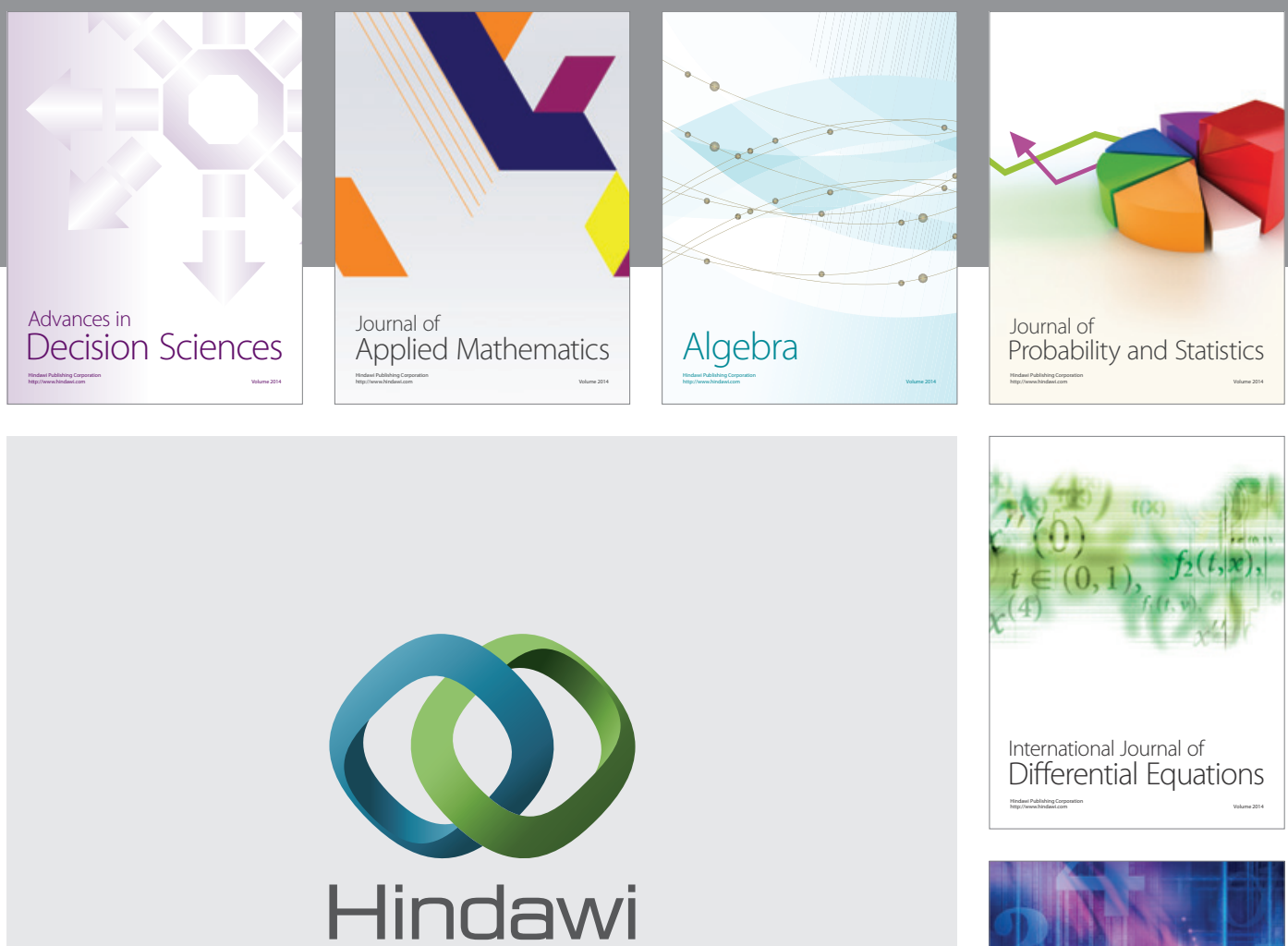

Submit your manuscripts at http://www.hindawi.com
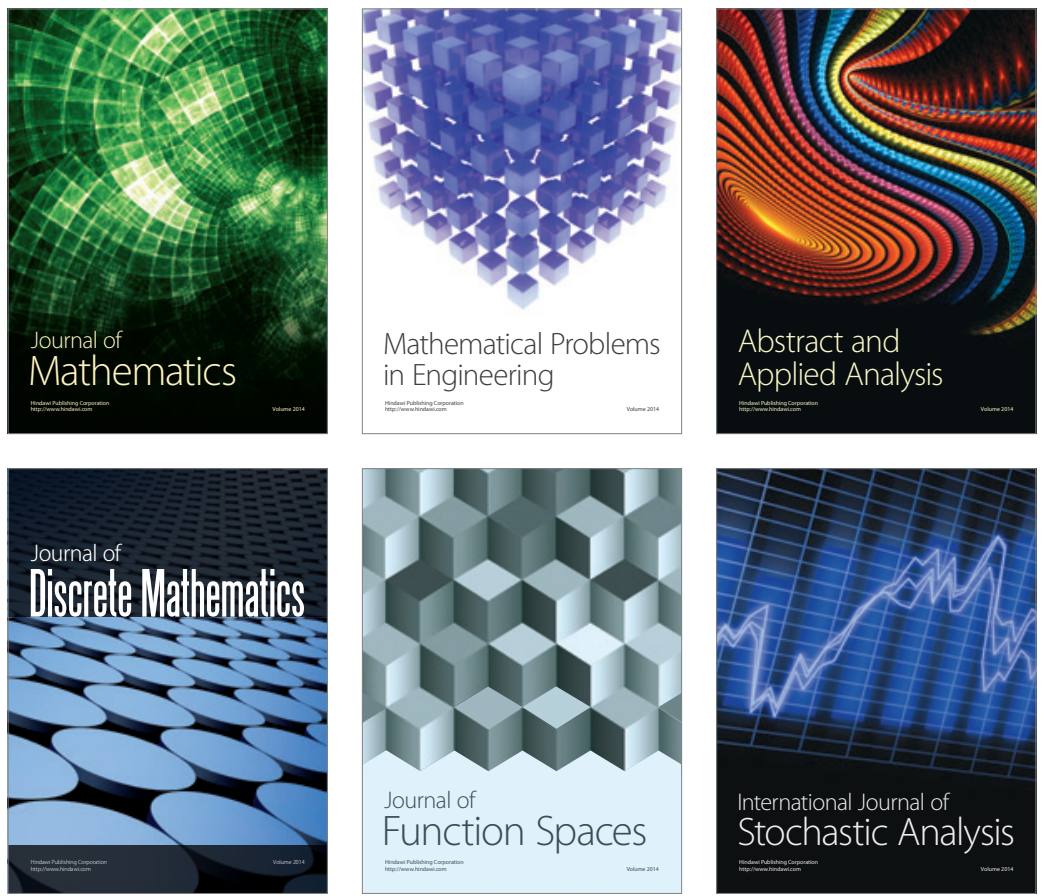

Journal of

Function Spaces

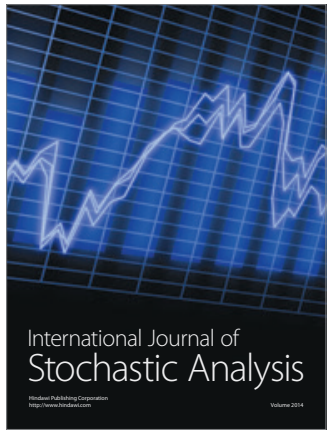

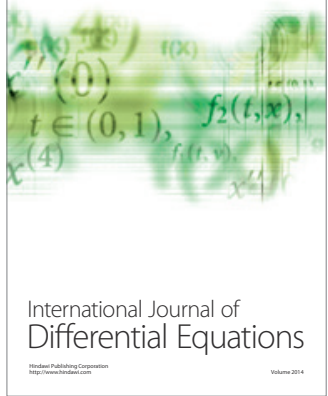
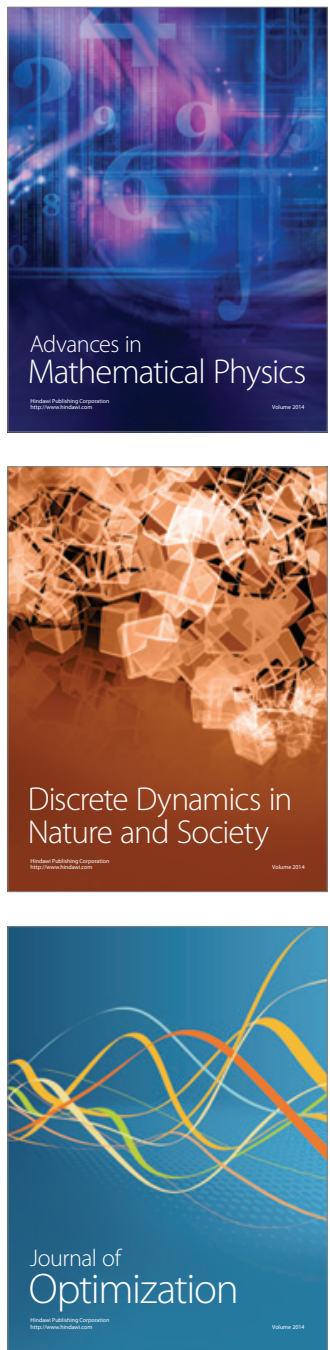